\title{
Éléments finis mixtes minimaux sur les polyèdres*
}

\author{
Snorre H. CHRISTIANSEN ${ }^{\dagger}$
}

12 juin 2009

\begin{abstract}
Résumé
Étant donné un complexe cellulaire constitué de polyèdres, plongé dans un espace Euclidien, nous construisons des espaces d'éléments finis de formes différentielles contenant celles qui sont polynomiales de degré maximal donné, ayant localement la propriété de suite exacte et d'extension, de telle sorte que parmi tous les espaces ayant ces trois propriétés, ils ont la plus petite dimension. Plus généralement nous construisons, pour tout système d'éléments finis inclus dans un système d'éléments finis compatible, un système d'éléments finis compatible intermédiaire et de dimension minimale.
\end{abstract}

\section{Cadre}

Soit $S$ un espace métrique compact. Une partie fermée $T$ de $S$ sera appellée cellule de dimension $l$ lorsqu'il existe une bijection bi-Lipschitzienne de $T$ sur la boule unité fermée $\mathbb{B}^{l}$ de $\mathbb{R}^{l}$. Le bord $\partial T$ de $T$ est alors par définition l'image réciproque de la spère $\mathbb{S}^{l-1}$, et l'intérieur de $T$ est $T \backslash \partial T$. La dimension est bien définie, et le bord ne dépend pas du choix de la bijection.

Un complexe cellulaire sur $S$ est un ensemble fini $\mathcal{T}$ de cellules dans $S$ tel que :

- La réunion de $\mathcal{T}$ est $S$, et $S$ porte la topologie induite.

- Deux cellules distinctes ont des intérieurs disjoints.

- La frontière de toute cellule est réunion de cellules.

La partie de $\mathcal{T}$ constituée des cellules de dimension $l$ sera notée $\mathcal{T}^{l}$.

Nous nous intéressons à la construction d'espaces de formes différentielles (FDs) sur $S$. Suivant [2][3] un système d'éléments finis $(\mathrm{SEF})$ sur $\mathcal{T}$ est la donnée pour chaque entier naturel $k$ et chaque $T \in \mathcal{T}$, d'un espace vectoriel $A^{k}(T)$ de $k$-FD sur $T$, de telle sorte que les deux propriétés suivantes soient satisfaites :

- La dérivée extérieure induit des applications d : $A^{k}(T) \rightarrow A^{k-1}(T)$.

- Si $T^{\prime} \subseteq T$ avec inclusion $i$, le retrotransport ("pullback") induit une application $i^{\star}: A^{k}(T) \rightarrow$ $A^{k}\left(T^{\prime}\right)$. Pour $u \in A^{k}(T)$ on notera $i^{\star} u=\left.u\right|_{T^{\prime}}$.

La trace sera dans la suite toujours entendue au sens du retrotransport par l'application inclusion.

Dans ces conditions nous notons, pour tout complexe cellulaire $\mathcal{T}^{\prime}$ inclus dans $\mathcal{T}$ :

$$
A^{k}\left(\mathcal{T}^{\prime}\right)=\left\{u=\left(u_{T}\right)_{T \in \mathcal{T}}: \forall T, T^{\prime} \in \mathcal{T} \quad u_{T} \in A^{k}(T) \text { et }\left.T^{\prime} \subseteq T \Rightarrow u_{T}\right|_{T^{\prime}}=u_{T^{\prime}}\right\} .
$$

Les éléments de $A^{k}(\mathcal{T})$ peuvent être identifiés à des FDs sur $S$ ayant juste assez de continuité aux interfaces entre cellules pour que la dérivée extérieure au sens des distributions (ou courants) corresponde à la dérivée extérieure prise par cellule.

Nous dirons qu'un SEF $A$ est compatible lorsque les deux conditions suivantes sont remplies :

*This work, conducted as part of the award "Numerical analysis and simulations of geometric wave equations" made under the European Heads of Research Councils and European Science Foundation EURYI (European Young Investigator) Awards scheme, was supported by funds from the Participating Organizations of EURYI and the EC Sixth Framework Program.

${ }^{\dagger}$ CMA, University of Oslo, PO Box 1053 Blindern, NO-0316 Oslo, Norway. email : snorrec@math.uio.no 
- Suite exacte. Pour toute cellule $T$, la suite suivante est exacte :

$$
0 \rightarrow \mathbb{R} \rightarrow A^{0}(T) \rightarrow A^{1}(T) \rightarrow \cdots \rightarrow A^{\operatorname{dim}(T)}(T) \rightarrow 0 .
$$

La deuxième flèche associe à un réel, la fonction sur $T$ prenant cette valeur uniquement.

- Extension. Pour toute cellule $T$, si $u \in A^{k}(\partial T)$, il existe au moins un $v \in A^{k}(T)$ dont la trace sur $\partial T$ est $u$.

On note $A_{0}^{k}(T)$ le sous-espace de $A^{k}(T)$ constitué des éléments ayant une trace nulle sur le bord.

Pour la démonstration des quatre propositions suivantes on pourra consulter [3].

Proposition 1.1. Pour un SEF A on a :

$$
\operatorname{dim} A^{k}(\mathcal{T}) \leq \sum_{T \in \mathcal{T}} \operatorname{dim} A_{0}^{k}(T),
$$

avec égalité lorsque la propriété d'extension est satisfaite.

On choisit une orientation pour chaque cellule, qui permet en particulier d'intégrer les formes de degré maximal. On note $C^{\bullet}(\mathcal{T})$ le complexe de cochaines associé a $\mathcal{T}$, muni de l'opération de cobord. Ainsi $C^{k}(\mathcal{T})=\mathbb{R}^{\mathcal{T}^{k}}$. L'application de De Rham $\mu$ est défini sur $A^{k}(\mathcal{T})$ par :

$$
\mu u=\left(\int_{T} u_{T}\right)_{T \in \mathcal{T}^{k}} \in C^{k}(\mathcal{T}) .
$$

Il s'agit d'un morphisme de complexes $A^{\bullet}(\mathcal{T}) \rightarrow C^{\bullet}(\mathcal{T})$, en conséquence du théorème de Stokes.

Proposition 1.2. Pour un SEF compatible, l'application de De Rham induit des isomorphismes en cohomologie.

Proposition 1.3. Pour un SEF A ayant la propriété d'extension, la propriété de suite exacte (2) est équivalente à la suivante (avec condition au bord homogène) :

- Pour toute cellule T, la suite suivante est exacte:

$$
0 \rightarrow A_{0}^{0}(T) \rightarrow A_{0}^{1}(T) \rightarrow \cdots \rightarrow A^{\operatorname{dim}(T)}(T) \rightarrow \mathbb{R} \rightarrow 0 .
$$

L'avant dernière flèche est l'intégration.

Proposition 1.4. Soit un SEF A dont chaque espace $A^{k}(T)$ est muni d'un produit scalaire noté a. On suppose que $T$ est une cellule telle que (5) est exacte. Pour chaque $\alpha \in \mathbb{R}$ il existe un unique élément u de $A^{\operatorname{dim} T}(T)$ tel que :

$$
\int_{T} u=\alpha \text { et } \forall v \in A_{0}^{\operatorname{dim} T-1}(T) \quad a(u, \mathrm{~d} v)=0 .
$$

Fixons $k<\operatorname{dim} T$. Tout $u \in A^{k}(\partial T)$ admettant une extension, admet une unique extension $u \in$ $A^{k}(T)$ telle que :

$$
\forall v \in A_{0}^{k}(T) \quad a(\mathrm{~d} u, \mathrm{~d} v)=0 \text { et } \forall v \in A_{0}^{k-1}(T) \quad a(u, \mathrm{~d} v)=0 .
$$

Un élément $u$ de $A^{k}(T)$ satisfaisant (7) sera dit harmonique. La proposition affirme donc l'existence et l'unicité d'une extension harmonique lorsqu'une extension existe. Si l'on a le choix entre plusieurs SEFs on parlera d'extension $A$-harmonique. L'orthogonalité pour $a$ sera notée $\perp$, de sorte que (7) s'écrit aussi :

$$
\mathrm{d} u \perp \mathrm{d} A_{0}^{k}(T) \text { et } u \perp \mathrm{d} A_{0}^{k-1}(T) .
$$




\section{Construction}

On suppose que $S$ est un domaine de $\mathbb{R}^{n}$ et que chaque cellule $T \in \mathcal{T}$ est un polyèdre. Pour $l<n$ les cellules de dimension $l$ ne sont pas nécessairement plats (càd. inclus dans un sous-espace affine de $\mathbb{R}^{n}$ de dimension $l$ ). Soit $p$ un entier naturel.

Pour tout $T \in \mathcal{T}$ et tout $k$, notons $A^{k}(T)$ les FDs sur $T$ qui sont trace sur $T$ d'une $k$-FD sur $\mathbb{R}^{n}$, polynomiale de degré au plus $p$. On note que lorsque $T \in \mathcal{T}^{l}$ n'est pas plat, la dimension de $A^{k}(T)$ peut être supérieure à la dimension de l'espace des $k$-formes polynomiales de degré $p$ sur $\mathbb{R}^{l}$. Nous avons bien là un SEF, mais il est loin d'être compatible.

Choisissons un raffinement de $\mathcal{T}$ qui soit un complexe simplicial - souvent le raffinement barycentrique convient. Notons $B^{k}(T)$ les $k$-FDs dites de Whitney sur $T$, relativement à ce raffinement et de degré $p+1$. Il s'agit de la première famille d'EF mixtes de Nédélec [5], généralisée aux FDs par Hiptmair [4], choisissant ceux compris entre les polynomes de degré $p$ et $p+1$. On pourra consulter [1] à leur sujet. Nous avons là un SEF compatible sur $\mathcal{T}$, mais il est affreusement grand.

Notre but est de construire un SEF compatible sur $\mathcal{T}$, intermédiaire entre $A$ et $B$ et aussi petit que possible. De fait nous en obtenons un qui est de dimension minimale sur chaque cellule et en tout degré, non seulement parmi ceux qui sont contenus dans $B$ mais en général.

Plus généralement nous supposons que $A$ est un SEF, que $B$ est un SEF compatible, et que $A^{k}(T) \subseteq B^{k}(T)$ pour tout $k$ et $T$ (voilà ce qu'on entend un peu abusivement par l'inclusion de $A$ dans $B$ ). La construction dépend du choix d'un produit scalaire $a$ sur chaque $B^{k}(T)$ (on pourra prendre si on veut le produit $\mathrm{L}^{2}$ sur les FDs). Elle se fait en deux étapes, la première complétant $A$ de sorte que la propriété de suite exacte - sous la forme (5) - soit satisfaite, la deuxième assurant la propriété d'extension, sans nuire aux acquis. Le SEF obtenu est alors compatible d'après la Proposition 1.3.

Première étape. Si $k<\operatorname{dim} T$, posons :

$$
E^{k}(T)=\left\{u \in B_{0}^{k}(T): \mathrm{d} u \in A_{0}^{k+1}(T), \mathrm{d} u \perp \mathrm{d} A_{0}^{k}(T) \text { et } u \perp \mathrm{d} B_{0}^{k-1}(T)\right\} .
$$

Dans le cas $k=\operatorname{dim} T$, si $A^{k}(T)$ contient un $u$ d'intégrale 1 , on pose $E^{k}(T)=0$, sinon on pose :

$$
E^{k}(T)=\left\{u \in B^{k}(T): u \perp \mathrm{d} B_{0}^{k-1}(T)\right\} .
$$

Remarquons que $A^{k}(T) \cap E^{k}(T)=0$ et posons :

$$
\tilde{A}^{k}(T)=A^{k}(T) \oplus E^{k}(T) .
$$

On note que $\mathrm{d}: E^{k}(T) \rightarrow \mathrm{H}^{k+1}\left(A_{0}^{\bullet}(T)\right.$ ) est un isomorphisme (si $k=\operatorname{dim} T$ interpréter la flèche comme l'intégration) ce qui donne en particulier :

$$
\operatorname{dim} E^{k}(T)=\operatorname{dim} \mathrm{H}^{k+1}\left(A_{0}^{\bullet}(T)\right) .
$$

Proposition 2.1. $\tilde{A}$ est un $S E F$ contenant $A$ tel que les suites :

$$
0 \rightarrow \tilde{A}_{0}^{0}(T) \rightarrow \tilde{A}_{0}^{1}(T) \rightarrow \cdots \rightarrow \tilde{A}^{\operatorname{dim}(T)}(T) \rightarrow \mathbb{R} \rightarrow 0,
$$

sont exactes.

Démonstration. Qu'il s'agisse d'un SEF contenant $A$ est immédiat. De plus :

$$
\tilde{A}_{0}^{k}(T)=A_{0}^{k}(T) \oplus E^{k}(T) .
$$

Supposons que $k<\operatorname{dim} T, u \in \tilde{A}_{0}^{k}(T)$ et $\mathrm{d} u=0$. Posons $u=v+w$ avec $v \in A_{0}^{k}(T)$ et $w \in E^{k}(T)$. On a $\mathrm{d} v+\mathrm{d} w=0$ et $\mathrm{d} v \perp \mathrm{d} w$, donc $\mathrm{d} v=0$ et $\mathrm{d} w=0$. Il s'ensuit que $w=0$.

Posons maintenant $v=x+y$ avec $x \in \mathrm{d} A_{0}^{k-1}(T)$ et $y \perp \mathrm{d} A_{0}^{k-1}(T)$. Puisque $\mathrm{d} y=0$ nous pouvons choisir $z \in B_{0}^{k-1}(T)$ tel que $\mathrm{d} z=y$ et $z \perp \mathrm{d} B_{0}^{k-2}(T)$. Alors $z \in E^{k-1}(T)$, ce qui, in fine, prouve que $u \in \mathrm{d} \tilde{A}_{0}^{k-1}(T)$.

Supposons maintenant $k=\operatorname{dim} T, u \in \tilde{A}^{k}(T)$ et $\int u=0$. Écrivons $u=v+w$ avec $v \in \mathrm{d} A_{0}^{k-1}(T)$ et $w \perp \mathrm{d} A_{0}^{k-1}(T)$. Choisissons $x \in B_{0}^{k-1}(T)$ tel que $\mathrm{d} x=w$ et $x \perp \mathrm{d} B_{0}^{k-2}(T)$. Alors $x \in E^{k-1}(T)$, donc $u \in \mathrm{d} \tilde{A}_{0}^{k-1}(T)$. 
Deuxième étape. Par abus on note $A$ le système $\tilde{A}$ qu'on vient de construire, ou plus généralement un SEF inclus dans $B$ ayant la proprieté (5) de suite exacte avec condition au bord homogène. Pour toute cellule $T$ nous allons compléter $A \bullet(T)$ pour assurer la propriété d'extension sur $T$ tout en préservant $A_{0}^{\bullet}(T)$. On commence par les cellules $T$ de dimension 1 , continue avec celles de dimension 2 et ainsi de suite.

Nous utilisons le résultat suivant où on note $\operatorname{tr}$ l'application trace $B^{\bullet}(T) \rightarrow B^{\bullet}(\partial T)$.

Proposition 2.2. Fixons un $k \leq l$. Tout $u \in B^{k}(\partial T)$ tel que $\mathrm{d} u \in \operatorname{tr} A^{k+1}(T)$ admet une unique extension $u \in B^{k}(T)$ telle que :

$$
\mathrm{d} u \in A^{k+1}(T), \mathrm{d} u \perp \mathrm{d} A_{0}^{k}(T) \text { et } u \perp \mathrm{d} B_{0}^{k-1}(T) .
$$

Démonstration. D'après la Proposition 1.2, la suite suivante est exacte :

$$
0 \rightarrow \mathbb{R} \rightarrow B^{0}(\partial T) \rightarrow B^{1}(\partial T) \rightarrow \cdots \rightarrow B^{l}(\partial T) \rightarrow \mathbb{R} \rightarrow 0
$$

Existence. On suppose d'abord $k<l$. Soit d'abord $v$ l'extension $A$-harmonique de $\mathrm{d} u$. Puisque $\mathrm{d} v$ est $A$-harmonique et nulle au bord on a $\mathrm{d} v=0$. Choisissons $u^{\prime} \in B^{k}(T)$ tel que $\mathrm{d} u^{\prime}=v$. On a $\mathrm{d}\left(\operatorname{tr} u^{\prime}-u\right)=0$, choisissons donc $u^{\prime \prime} \in B^{k-1}(T)$ tel que $\mathrm{d} \operatorname{tr} u^{\prime \prime}=\operatorname{tr} u^{\prime}-u$. Alors $u^{\prime}-\mathrm{d} u^{\prime \prime}$ est une extension de $u$ de dérivée extérieure $v$. Par ajout d'un élément $\mathrm{de} \mathrm{d} B_{0}^{k-1}(T)$ on assure aussi son orthogonalité à $\mathrm{d} B_{0}^{k-1}(T)$.

Supposons maintenant $k=l$. Soit $v \in A^{k+1}(T)$ tel que $\int v=\int u$ et $v \perp \mathrm{d} A_{0}^{k}(T)$. Soit $u^{\prime} \in B^{k}(T)$ tel que $\mathrm{d} u^{\prime}=v$. Par Stokes on a $\int\left(\operatorname{tr} u^{\prime}-u\right)=0$, choisissons donc $u^{\prime \prime} \in B^{k-1}(T)$ tel que $\mathrm{d} \operatorname{tr} u^{\prime \prime}=\operatorname{tr} u^{\prime}-u$. Alors $u^{\prime}-\mathrm{d} u^{\prime \prime}$ est comme avant une extension de $u$ de dérivée extérieure $v$ et par ajout d'un élément de $\mathrm{d} B_{0}^{k-1}(T)$ on assure aussi son orthogonalité à $\mathrm{d} B_{0}^{k-1}(T)$.

Unicité. Si $u \in B_{0}^{k}(T)$ satisfait (15), alors $\mathrm{d} u=0$ et $u \perp \mathrm{d} B_{0}^{k-1}(T)$ ce qui donne $u=0$.

Supposons que nous avons rempli notre programme pour les cellules de dimension au plus $l$ $(l \geq 0)$, le SEF étendu étant noté $\tilde{A}$. On considère une cellule $T$ de dimension $l+1$.

Pour $k \leq l$ posons :

$$
F^{k}=\left\{u \in \tilde{A}^{k}(\partial T): u \perp \operatorname{tr} A^{k}(T) \text { et } \mathrm{d} u \in \operatorname{tr} A^{k+1}(T)\right\}
$$

et notons $\tilde{F}^{k}$ les extensions des éléments de $F^{k}$ définies par la Proposition 2.2 ci-dessus. On pose aussi :

$$
G^{k}=\left\{u \in \tilde{A}^{k}(\partial T): \mathrm{d} u \perp \operatorname{tr} A^{k+1}(T) \text { et } u \perp\left\{v \in \tilde{A}^{k}(\partial T): \mathrm{d} u=0\right\}\right\},
$$

et on note $\tilde{G}^{k}$ les extensions $B$-harmoniques des éléments de $G^{k}$. Finalement on pose :

$$
\tilde{A}^{k}(T)=A^{k}(T)+\tilde{F}^{k}+\tilde{G}^{k} .
$$

On remarque que $\mathrm{d} \tilde{F}^{k} \subseteq A^{k+1}(T)$. Si $k<l$, $\mathrm{d} \tilde{G}^{k} \subseteq \tilde{F}^{k+1}(T)$ et si $k=l, \tilde{G}^{k}=0$. On a une décomposition en somme directe :

$$
\tilde{A}^{k}(\partial T)=\operatorname{tr} A^{k}(T) \oplus F^{k} \oplus G^{k} .
$$

Il s'ensuit en particulier que $\operatorname{tr}: \tilde{A}^{k}(T) \rightarrow \tilde{A}^{k}(\partial T)$ est surjectif. De plus, si $u \in A^{k}(T), v \in \tilde{F}^{k}$ et $w \in \tilde{G}^{k}$ satisfont $\operatorname{tr}(u+v+w)=0$, alors $\operatorname{tr} v=0$ et $\operatorname{tr} w=0$, ce qui donne $v=0$ et $w=0$. Si en fait $u+v+w=0$ on aura aussi $u=0$. Ainsi la somme (19) est directe et :

$$
\tilde{A}_{0}^{k}(T)=A_{0}^{k}(T) \text {. }
$$

Pour $k=l+1$ on prend simplement $\tilde{A}^{k}(T)=A^{k}(T)$. Ceci achève la construction ainsi que sa justification, en dimension $l+1$.

Pour finir nous notons que si $\hat{A}$ est un quelconque SEF compatible contenant $A$ on a :

$$
\operatorname{dim} \hat{A}_{0}^{k}(T) \geq \operatorname{dim} A_{0}^{k}(T)+\operatorname{dim} \mathrm{H}^{k+1}\left(A_{0}^{\bullet}(T)\right),
$$

puisque $\hat{A}$ permet de construire des espaces $\tilde{A}_{0}^{k}(T) \subseteq \hat{A}_{0}^{k}(T)$ tels que (12) et (14). La construction proposée donne l'égalité dans (22) et donc la plus petite dimension possible d'après la Proposition 1.1 . 


\section{Références}

[1] D. N. Arnold, R. S. Falk, and R. Winther. Finite element exterior calculus, homological techniques, and applications. Acta Numer., 15 :1-155, 2006.

[2] S. H. Christiansen. A construction of spaces of compatible differential forms on cellular complexes. Math. Models Methods Appl. Sci., 18(5) :739-757, 2008.

[3] S. H. Christiansen. Foundations of finite element methods for wave equations of Maxwell type. Department of Mathematics, University of Oslo, E-print, 5, 2009.

[4] R. Hiptmair. Canonical construction of finite elements. Math. Comp., 68(228) :1325-1346, 1999.

[5] J.-C. Nédélec. Mixed finite elements in $\mathbf{R}^{3}$. Numer. Math., 35(3) :315-341, 1980. 